\title{
Cooperative Strategies and Achievable Rates for Two User OFDMA Channels
}

\author{
Sezi Bakım and Onur Kaya, Member, IEEE
}

\begin{abstract}
We propose three encoding strategies for a two user cooperative Orthogonal Frequency Division Multiple Access (OFDMA) system, based on block Markov superposition encoding (BMSE). We obtain the expressions for the resulting achievable rate regions for all three encoding strategies. We show that, by allowing for re-partitioning and re-encoding of the cooperative messages across subchannels, it is possible to better exploit the diversity created by OFDMA, and higher rates can be achieved. We demonstrate potential rate gains attained by cooperative OFDMA, through simulations.
\end{abstract}

Index Terms-Block Markov encoding, diversity, OFDMA, user cooperation.

\section{INTRODUCTION}

$\mathbf{O}$ FDMA is a multiple accessing technique which provides a relatively simple way of assigning available bandwidth to users, while avoiding interference. Furthermore, it converts a frequency selective fading channel, into parallel flat fading subchannels, hence reducing the effects of intersymbol interference. However, like all orthogonal multiple accessing techniques, OFDMA incurs a loss in achievable rates. Moreover, in wireless channels, what is considered as interference is in fact side information, and combined with the diversity created by the orthogonal subchannels in OFDMA, this side information can be exploited to increase the rates achievable by the users.

The idea of mutual cooperation in wireless channels roots from the abstract model, known as multiple access channel (MAC) with generalized feedback, introduced in [2], [3]. In [3], an achievable rate region, which was larger than that of [2], was obtained by utilizing BMSE and backward decoding. More recently, in [4], the MAC with generalized feedback was used to model a fading cooperative additive white Gaussian noise (AWGN) channel, and the results therein made cooperative communications very attractive.

The use of cooperative protocols in OFDM systems was investigated extensively by many authors over the recent years. In [5], the authors obtained bounds on pairwise error probability for single antenna OFDM systems employing cooperative convolutional codes. In [6], per subcarrier hybrid cooperation strategies were proposed, with the goal of minimizing the error probability. Methods for subcarrier selection in multihop

Manuscript received December 31, 2010; revised June 30, 2011; accepted September 23, 2011. The associate editor coordinating the review of this letter and approving it for publication was G. Yue.

The authors are with the Department of Electrical and Electronics Engineering, Işık University, Istanbul, Turkey (e-mail: sezi.bakim@isik.edu.tr, onurkaya@isikun.edu.tr).

This work was presented in part at the IEEE Global Communications Conference, Miami, FL, Dec 2010 [1], and was supported by The Scientific \& Technological Research Council of Turkey, Grant 108E208.

Digital Object Identifier 10.1109/TWC.2011.101811.102334
OFDM systems were developed in [7]. Resource allocation for an OFDMA system where each user is allowed to transmit and receive at the same time, but necessarily on different subcarriers, was considered in [8]. An amplify-and-forward (AF) scheme based on time-division duplex transmissions was employed in [9] with the aim of maximizing system throughput and enhancing fairness in a cooperative OFDMA uplink system.

The works in the literature related to cooperative OFDM, some examples of which are listed above, either consider a one sided cooperation strategy, or a mutually cooperative strategy based on two parallel dedicated relay channels, or mutual cooperation based on a time division protocol. In this paper, without imposing any prior constraints on which users will use which subchannels, we first propose two full-duplex cooperative encoding strategies: intra-subchannel and intersubchannel cooperative encoding. These strategies use decode and forward (DF) approach and are based on BMSE. Intrasubchannel cooperative encoding is an extension of the two user cooperative strategy in [3] and [4] to OFDMA, and intersubchannel cooperative encoding is a novel method which allows for re-partitioning and re-encoding of the cooperative messages across subchannels. We further propose a more practical encoding technique with half-duplex operation in each subchannel, and call it half-duplex cooperative encoding. We obtain the achievable rate regions for all three strategies, and compare them with the non-cooperative OFDMA capacity region through simulations, which demonstrate promising rate improvements.

\section{System Model}

We consider a fading two user cooperative OFDMA system with $N$ subchannels. On each subchannel, unless otherwise stated, each user is capable of both transmitting and receiving signals. The system is illustrated in Figure 1, and is modelled by,

$$
\begin{aligned}
& Y_{0}^{(i)}=h_{10}^{(i)} X_{1}^{(i)}+h_{20}^{(i)} X_{2}^{(i)}+Z_{0}^{(i)}, \\
& Y_{1}^{(i)}=h_{21}^{(i)} X_{2}^{(i)}+Z_{1}^{(i)}, \\
& Y_{2}^{(i)}=h_{12}^{(i)} X_{1}^{(i)}+Z_{2}^{(i)},
\end{aligned}
$$

where, for each subchannel $i \in\{1, \ldots, N\}, X_{k}^{(i)}$ is the symbol transmitted by node $k, Z_{j}^{(i)}$ is the zero-mean additive white Gaussian noise at node $j, h_{k j}^{(i)}$ is the instantaneous fading coefficient between nodes $k$ and $j$, known to all nodes, and $Y_{j}^{(i)}$ is the symbol received at node $j$; with $k \in\{1,2\}$, $j \in\{0,1,2\}$ and $k \neq j$. Here, the receiver is denoted by $j=0$. The variance of $Z_{j}^{(i)}$ is given by $\sigma_{j}^{(i)^{2}}$. To simplify 


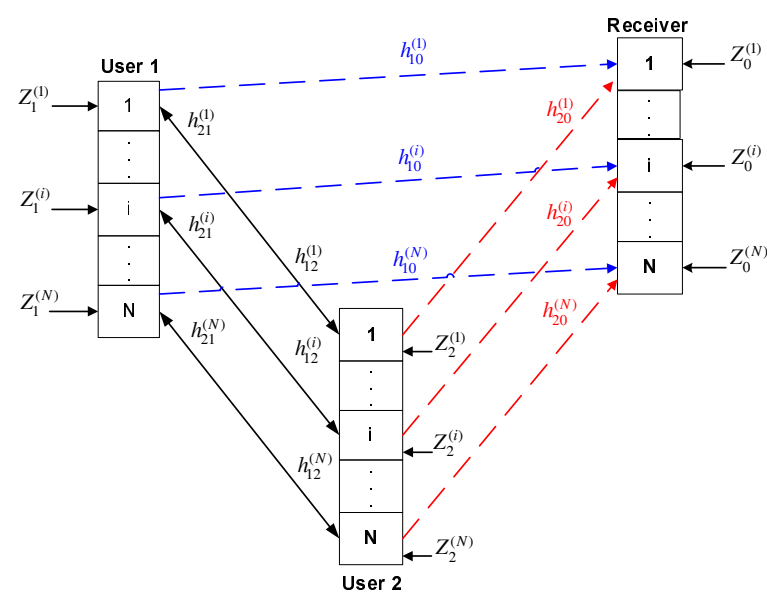

Fig. 1. Gaussian cooperative OFDMA channel model.

the notation throughout the paper, we define the normalized power-fading coefficients $s_{k j}^{(i)}=\frac{\left(h_{k j}^{(i)}\right)^{2}}{\sigma_{j}^{(i)^{2}}}$, and the Gaussian capacity function, $C(x) \triangleq \frac{1}{2} \log (1+x)$.

\section{Coding Techniques And RATE REgions FOR COOPERATIVE OFDMA}

\section{A. Intra-subchannel Cooperative Encoding vs Inter- subchannel Cooperative Encoding}

The channel model given in (1)-(3) consists of $N$ orthogonal two user cooperative multiple access channels (CMAC) in parallel, over which users 1 and 2 want to convey two independent messages, $w_{1}$ and $w_{2}$ respectively. Therefore, the encoding and decoding techniques known for the two user CMAC [4] can be easily extended to this system, by splitting the total message to be transmitted into smaller submessages, and encoding each submessage independently over the orthogonal cooperative channels. This approach, which we call intra-subchannel cooperative encoding, first divides the messages into two submessages, i.e., $w_{1}=\left\{w_{10}, w_{12}\right\}$ and $w_{2}=\left\{w_{20}, w_{21}\right\}$, as in [3], [4]. Here, $w_{k j}$ is intended to be decoded by user $j$ and the receiver, while $w_{k 0}$ is intended only for the receiver, where $j, k \in\{1,2\}$ and $j \neq k$. These two submessages are further divided into $N$ submessages each, to be separately transmitted over $N$ subchannels,

$$
w_{k 0}=\left\{w_{k 0}^{(1)}, \ldots, w_{k 0}^{(N)}\right\}, \quad w_{k j}=\left\{w_{k j}^{(1)}, \ldots, w_{k j}^{(N)}\right\},
$$

with the respective rates $\left\{R_{k 0}^{(1)}, \ldots, R_{k 0}^{(N)}\right\}$ and $\left\{R_{k j}^{(1)}, \ldots, R_{k j}^{(N)}\right\}$, and are encoded separately over each subchannel $i=1, \cdots, N$, by using a straightforward extension of BMSE [3], [4], to OFDMA,

$$
\begin{aligned}
X_{k}^{(i)}= & \sqrt{p_{k 0}^{(i)}} X_{k 0}^{(i)}\left(w_{k 0}^{(i)}[b], U^{(i)}\left(w_{k j}^{(i)}[b-1], \hat{w}_{j k}^{(i)}[b-1]\right)\right) \\
& +\sqrt{p_{k j}^{(i)}} X_{k j}^{(i)}\left(w_{k j}^{(i)}[b], U^{(i)}\left(w_{k j}^{(i)}[b-1], \hat{w}_{j k}^{(i)}[b-1]\right)\right) \\
& +\sqrt{p_{U_{k}}^{(i)}} U_{k}^{(i)}\left(w_{k j}^{(i)}[b-1], \hat{w}_{j k}^{(i)}[b-1]\right)
\end{aligned}
$$

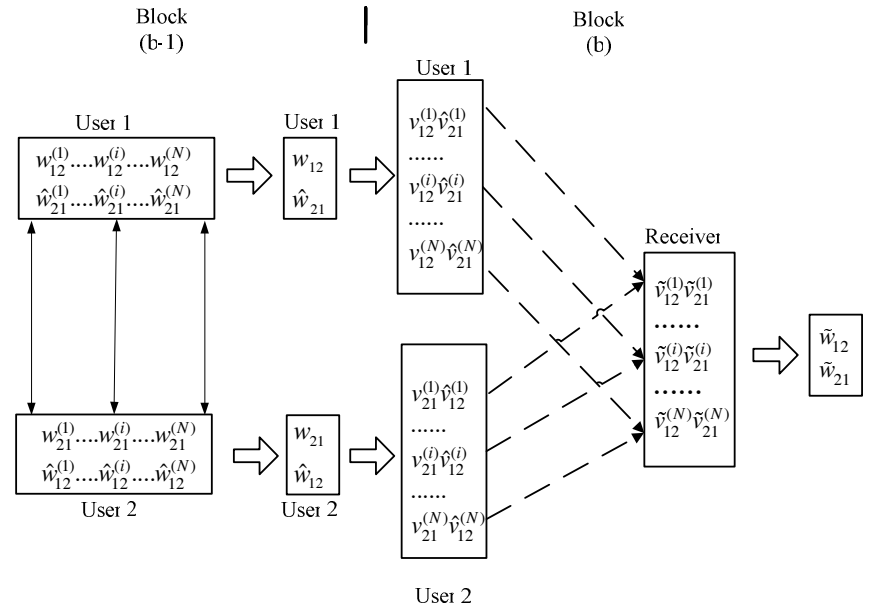

Fig. 2. Re-partitioning of cooperative messages in inter-subchannel cooperative encoding. For the ease of demostration, the transmission to the receiver in block $b-1$, and the transmissions among the users in block $b$ are not shown.

with the key difference that the powers assigned to each codeword in (5), are required to satisfy the sum power constraints over each subchannel,

$$
\sum_{i} p_{k 0}^{(i)}+p_{k j}^{(i)}+p_{U_{k}}^{(i)}=\sum_{i} p_{k}^{(i)} \leq \bar{p}_{k} .
$$

where $k, j \in\{1,2\}$ and $j \neq k$. In (5), $X_{k 0}^{(i)}$ carries the fresh information intended for the receiver, $X_{k j}^{(i)}$ carries the information intended for transmitter $j$ for cooperation in the next block and $U_{k}^{(i)}$ is the common information sent by both transmitters for the resolution of the remaining uncertainty from the previous block, all of which are transmitted over the ith subchannel and chosen from unit Gaussian distributions. The caret, as in $\hat{w}_{j k}^{(i)}[b-1]$, will be used to denote the estimates of messages at the cooperative partner throughout the paper. Note that $U_{1}^{(i)}=U_{2}^{(i)}=U^{(i)}$, when $\hat{w}_{k j}^{(i)}[b-1]=w_{k j}^{(i)}[b-1]$.

The drawback of the intra-subchannel cooperative encoding is that, it does not allow us to take full advantage of the diversity created by OFDMA across subchannels: if on one subchannel, the inter-user link is very strong, but userdestination links are consistently very weak, the total data rate of the submessage on that link will be dictated by the weaker link. If, however, we allow submessages received by a user on each subchannel to be combined, re-partitioned and re-allocated onto the subchannels, we will potentially obtain better rates. Therefore, we propose an implementation of such an approach, and call it inter-subchannel cooperative encoding.

In inter-subchannel cooperative encoding, since user $j$ 's real goal is to decode and re-encode the whole message $w_{k j}$ of user $k$, in re-encoding each $w_{k j}$ can be divided into new submessages,

$$
w_{12}=\left\{v_{12}^{(1)}, \ldots, v_{12}^{(N)}\right\}, \quad w_{21}=\left\{v_{21}^{(1)}, \ldots, v_{21}^{(N)}\right\},
$$

with the new respective rates $\left\{R_{12}^{\prime(1)}, \ldots, R_{12}^{\prime(N)}\right\}$ and $\left\{R_{21}^{\prime(1)}, \ldots\right.$, $\left.R_{21}^{\prime(N)}\right\}$. The exchange, re-partitioning, re-transmission and decoding of cooperative messages is illustrated in Figure 2. It is assumed that a table to match the new cooperative 
submessages, $v_{k j}^{(i)}$, to those received in the previous block, $w_{k j}^{(i)}$, is available at the users and the receiver. Hence, the receiver can obtain $w_{k j}$ upon decoding $v_{k j}^{(i)}$ for all $i$.

It is important to note that, since $\left\{w_{k j}^{(i)}\right\}_{i=1}^{N}$ and $\left\{v_{k j}^{(i)}\right\}_{i=1}^{N}$, are just different partitionings of the same message $w_{k j}$, their total rates have to be the same, i.e.,

$$
\begin{aligned}
& 2^{n R_{12}}=2^{n R_{12}^{(1)}+\ldots+n R_{12}^{(N)}}=2^{n R_{12}^{\prime(1)}+\ldots+n R_{12}^{\prime(N)}}, \\
& 2^{n R_{21}}=2^{n R_{21}^{(1)}+\ldots+n R_{21}^{(N)}}=2^{n R_{21}^{\prime(1)}+\ldots+n R_{21}^{\prime(N)}} .
\end{aligned}
$$

The way BMSE is executed is mostly similar to the case of intra-subchannel cooperative encoding, with the key difference that, in block $b$, the cooperative codewords $U_{k}^{(i)}$ of each user $k$ are now assigned to $\hat{v}_{j k}^{(i)}[b-1]$ and $v_{k j}^{(i)}[b-1]$, which are re-partitionings of the cooperative messages exchanged in the previous block. Then, in each block $b=1, \cdots, B$, the codewords transmitted by user $k$, where $j, k \in\{1,2\}, k \neq j$, $\forall i \in\{1, \cdots, N\}$ are formed using

$$
\begin{aligned}
X_{k}^{(i)}= & \sqrt{p_{k 0}^{(i)}} X_{k 0}^{(i)}\left(w_{k 0}^{(i)}[b], U^{(i)}\left(v_{k j}^{(i)}[b-1], \hat{v}_{j k}^{(i)}[b-1]\right)\right) \\
& +\sqrt{p_{k j}^{(i)}} X_{k j}^{(i)}\left(w_{k j}^{(i)}[b], U^{(i)}\left(v_{k j}^{(i)}[b-1], \hat{v}_{j k}^{(i)}[b-1]\right)\right) \\
& +\sqrt{p_{U_{k}}^{(i)}} U_{k}^{(i)}\left(v_{k j}^{(i)}[b-1], \hat{v}_{j k}^{(i)}[b-1]\right),
\end{aligned}
$$

where the powers still satisfy (6). Having described both encoding strategies, we are now ready to state our main result:

Theorem 1: For a two user cooperative OFDMA channel, define the rate constraints,

$$
\begin{gathered}
R_{12}^{(i)}<C_{12}^{(i)} \triangleq E\left[C\left(\frac{s_{12}^{(i)} p_{12}^{(i)}}{s_{12}^{(i)} p_{10}^{(i)}+1}\right)\right], \\
R_{21}^{(i)}<C_{21}^{(i)} \triangleq E\left[C\left(\frac{s_{21}^{(i)} p_{21}^{(i)}}{s_{21}^{(i)} p_{20}^{(i)}+1}\right)\right], \\
R_{10}^{(i)}<C_{10}^{(i)} \triangleq E\left[C\left(s_{10}^{(i)} p_{10}^{(i)}\right)\right], \\
R_{20}^{(i)}<C_{20}^{(i)} \triangleq E\left[C\left(s_{20}^{(i)} p_{20}^{(i)}\right)\right], \\
R_{10}^{(i)}+R_{20}^{(i)}<C_{0}^{(i)} \triangleq E\left[C\left(s_{10}^{(i)} p_{10}^{(i)}+s_{20}^{(i)} p_{20}^{(i)}\right)\right], \\
R_{12}^{(i)}+R_{21}^{(i)}+R_{10}^{(i)}+R_{20}^{(i)}<C_{s}^{(i)}, \\
R_{12}^{(i)}+R_{21}^{(i)}+R_{10}^{(i)}+R_{20}^{(i)}<C_{s}^{(i)},
\end{gathered}
$$

where

$$
C_{s}^{(i)} \triangleq E\left[C\left(s_{10}^{(i)} p_{1}^{(i)}+s_{20}^{(i)} p_{2}^{(i)}+2 \sqrt{s_{10}^{(i)} s_{20}^{(i)} p_{U_{1}}^{(i)} p_{U_{2}}^{(i)}}\right)\right],
$$

and

$$
\begin{aligned}
& R_{1}=\sum_{i=1}^{N} R_{1}^{(i)}=\sum_{i=1}^{N} R_{10}^{(i)}+R_{12}^{(i)}=\sum_{i=1}^{N} R_{10}^{(i)}+R_{12}^{(i)}, \\
& R_{2}=\sum_{i=1}^{N} R_{2}^{(i)}=\sum_{i=1}^{N} R_{20}^{(i)}+R_{21}^{(i)}=\sum_{i=1}^{N} R_{20}^{(i)}+R_{21}^{\prime(i)} .
\end{aligned}
$$

(a) Using intra-subchannel cooperative encoding, the closure of the convex hull of all rate pairs $\left(R_{1}, R_{2}\right)$ satisfying (10)-
(15), or equivalently

$$
\begin{aligned}
R_{1} & <\sum_{i} \min \left\{C_{12}^{(i)}+C_{10}^{(i)}, C_{s}^{(i)}\right\}, \\
R_{2} & <\sum_{i} \min \left\{C_{21}^{(i)}+C_{20}^{(i)}, C_{s}^{(i)}\right\}, \\
R_{1}+ & R_{2}<\sum_{i} \min \left\{C_{12}^{(i)}+C_{21}^{(i)}+C_{0}^{(i)}, C_{s}^{(i)}\right\},
\end{aligned}
$$

are achievable, where the convex hull is taken over all power allocation policies that satisfy (6).

(b) Using inter-subchannel cooperative encoding, the closure of the convex hull of all rate pairs $\left(R_{1}, R_{2}\right)$ satisfying (10)(14), (16), or equivalently

$$
\begin{aligned}
R_{1} & <\sum_{i} C_{12}^{(i)}+C_{10}^{(i)}, \\
R_{2} & <\sum_{i} C_{21}^{(i)}+C_{20}^{(i)}, \\
R_{1}+R_{2} & <\min \left\{\sum_{i} C_{12}^{(i)}+C_{21}^{(i)}+C_{0}^{(i)}, \sum_{i} C_{s}^{(i)}\right\},
\end{aligned}
$$

are achievable, where the convex hull is taken over all power allocation policies that satisfy (6).

Proof: Here, we provide an outline of the achievability proof; a more complete version including the probability of error analysis can be found in [10]. The proof for part (a) follows from a suitable extension of the proofs in [3], [4], to $N$ parallel subchannels; only the transition from (10)-(15) to (20)-(22) requires some attention: the rates of submessages $w_{12}^{(i)}$ and $w_{21}^{(i)}$ are subject to two constraints, one for the interuser link and one for the user-receiver links. Therefore, the rate $R_{k}^{(i)}$ for user $k$ on each subchannel $i$ is separately restricted by the minimum of $C_{k j}^{(i)}+C_{k 0}^{(i)}$ and $C_{s}^{(i)}$, hence the need for the extra minimum operations on single user rates.

The proof of part (b), whose steps are summarized in Table I, proceeds as follows: in each block $b$, and on each subchannel $i$, each user $j$ uses joint typicality check to decode $\hat{w}_{k j}^{(i)}(b)$, using $X_{k j}^{(i)}$, and treating $X_{k 0}^{(i)}$ as noise, leading to the constraints (26), (27) in Table I. The receiver on the other hand uses backwards decoding [3] to determine the transmitted messages. That is, in block $B$, using all received codewords, the receiver decodes $\tilde{v}_{12}^{(i)}[B-1]$ and $\tilde{v}_{21}^{(i)}[B-1]$ over each subchannel $i$ using joint typicality check, and therefore also knows $\tilde{w}_{12}[B-1]$ and $\tilde{w}_{21}[B-1]$. Then, in block $B-1$, it uses this information to jointly decode $\left\{\tilde{v}_{12}^{(i)}[B-2], \tilde{w}_{10}^{(i)}[B-1], \tilde{v}_{21}^{(i)}[B-2], \tilde{w}_{20}^{(i)}[B-1]\right\}$, based on which it may also deduce $\tilde{w}_{12}[B-2]$ and $\tilde{w}_{21}[B-2]$, and this process continues until the first block. The estimates of the repartitioned cooperative messages $\tilde{v}_{k j}^{(i)}[b-1]$ are converted to estimates of the cooperative messages $\tilde{w}_{k j}^{(i)}[b-1]$ using the match-up table available at the users and the receiver. Using this decoding strategy, and well known properties of jointly typical sequences [3], [11], it can be shown that (28)-(31) are sufficient to guarantee asymptotically error free decoding at the receiver. Finally, evaluating (26)-(31) for the generated Gaussian codewords, we obtain (10)-(14), (16).

The equivalence of constraints (23)-(25) to (10)-(14), (16) can be proved as follows: (23)-(24) follow trivially from (10)- 
TABLE I

THE SKETCH OF ACHIEVABILITY PROOF.

\begin{tabular}{|c|c|c|c|}
\hline 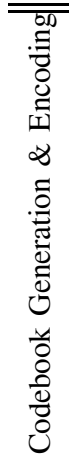 & \multicolumn{3}{|c|}{ 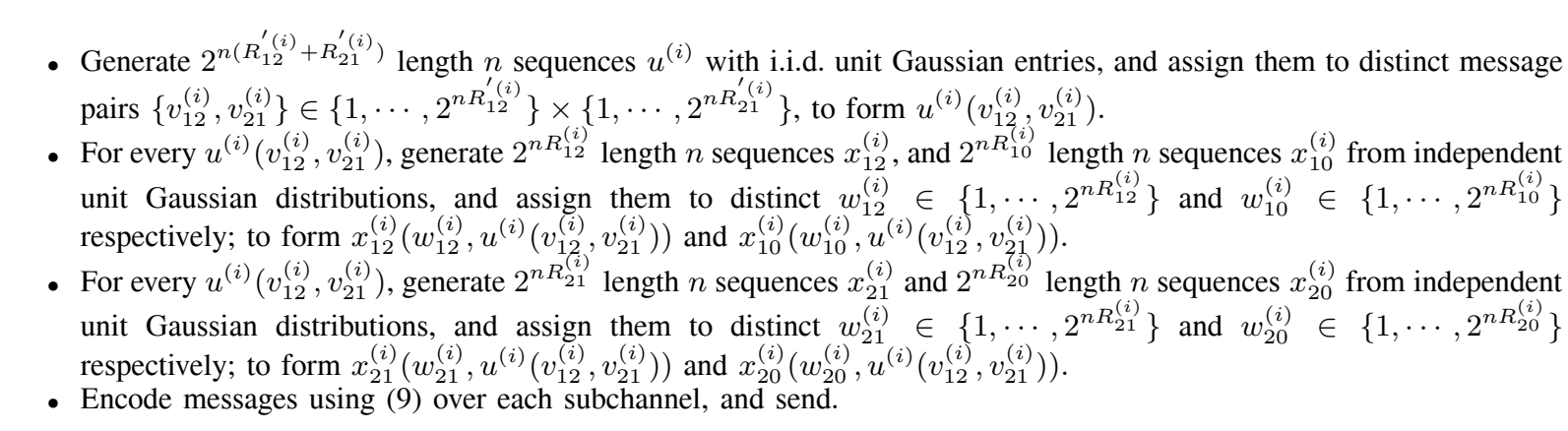 } \\
\hline & ing Rule & $\overline{\text { Resulting Rate Constraints for Reliable D }}$ & \\
\hline & $\begin{array}{l}\text { Find } \hat{w}_{k j}^{(i)}[b] \text { s.t. } \\
\left\{y_{j}^{(i)}[b], u^{(i)}\left(\hat{v}_{k j}^{(i)}[b-1], v_{j k}^{(i)}[b-1]\right),\right. \\
x_{k j}^{(i)}\left(\hat{w}_{k j}^{(i)}[b], u^{(i)}\left(\hat{v}_{k j}^{(i)}[b-1], v_{j k}^{(i)}[b-1]\right)\right), \\
x_{j k}^{(i)}\left(w_{j k}^{(i)}[b], u^{(i)}\left(\hat{v}_{k j}^{(i)}[b-1], v_{j k}^{(i)}[b-1]\right)\right), \\
\left.x_{j}^{(i)}\left(w_{j 0}^{(i)}[b], w_{j k}^{(i)}[b], u^{(i)}\left(\hat{v}_{k j}^{(i)}[b-1], v_{j k}^{(i)}[b-1]\right)\right)\right\} \\
\text { are jointly typical. }\end{array}$ & $\begin{array}{l}R_{12}^{(i)}<I\left(X_{12}^{(i)} ; Y_{2}^{(i)} \mid X_{2}^{(i)}, U^{(i)}\right) \\
R_{21}^{(i)}<I\left(X_{21}^{(i)} ; Y_{1}^{(i)} \mid X_{1}^{(i)}, U^{(i)}\right)\end{array}$ & $\begin{array}{l}(26) \\
(27)\end{array}$ \\
\hline 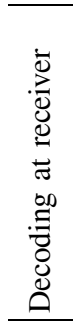 & $\begin{array}{l}\text { Find } \tilde{v}_{12}^{(i)}[b-1], \tilde{v}_{21}^{(i)}[b-1], \tilde{w}_{10}^{(i)}[b], \tilde{w}_{20}^{(i)}[b] \text { s.t. } \\
\left\{y^{(i)}[b], u^{(i)}\left(\tilde{v}_{12}^{(i)}[b-1], \tilde{v}_{21}^{(i)}[b-1]\right),\right. \\
x_{12}^{(i)}\left(\tilde{w}_{12}^{(i)}[b], u^{(i)}\left(\tilde{v}_{12}^{(i)}[b-1], \tilde{v}_{21}^{(i)}[b-1]\right)\right), \\
x_{21}^{(i)}\left(\tilde{w}_{21}^{(i)}[b], u^{(i)}\left(\tilde{v}_{12}^{(i)}[b-1] \tilde{v}_{21}^{(i)}[b-1]\right)\right), \\
x_{1}^{(i)}\left(\tilde{w}_{10}^{(i)}[b], \tilde{w}_{12}^{(i)}[b], u^{(i)}\left(\tilde{v}_{12}^{(i)}[b-1], \tilde{v}_{21}^{(i)}[b-1]\right)\right), \\
\left.x_{2}^{(i)}\left(\tilde{w}_{20}^{(i)}[b], \tilde{w}_{21}^{(i)}[b], u^{(i)}\left(\tilde{v}_{12}^{(i)}[b-1], \tilde{v}_{21}^{(i)}[b-1]\right)\right)\right\} \\
\text { are jointly typical. }\end{array}$ & $\begin{aligned} R_{10}^{(i)} & <I\left(X_{1}^{(i)} ; Y^{(i)} \mid X_{2}^{(i)}, X_{12}^{(i)}, U^{(i)}\right), \\
R_{20}^{(i)} & <I\left(X_{2}^{(i)} ; Y^{(i)} \mid X_{1}^{(i)}, X_{21}^{(i)}, U^{(i)}\right), \\
R_{10}^{(i)}+R_{20}^{(i)} & <I\left(X_{1}^{(i)}, X_{2}^{(i)} ; Y^{(i)} \mid X_{12}^{(i)}, X_{21}^{(i)}, U^{(i)}\right), \\
R_{12}^{\prime(i)}+R_{21}^{(i)} & +R_{10}^{(i)}+R_{20}^{(i)}<I\left(X_{1}^{(i)}, X_{2}^{(i)} ; Y^{(i)}\right) .\end{aligned}$ & $\begin{array}{l}(28) \\
(29) \\
(30) \\
(31)\end{array}$ \\
\hline
\end{tabular}

(13) and (18)-(19). The constraint (25) follows from (10)-(11), (14), (16) and the fact that $R_{k j}^{(i)}$ and $R_{k j}^{\prime(i)}$ are constrained separately, and need not be equal on a given subchannel $i$; as long as their sum over all subchannels remains the same.

Remark: Comparing the achievable rate region (23)-(25) with (20)-(22), we see that the minimum operations required for the individual rate constraints for each subchannel are removed, and the minimum operation required for the sum rate constraint (22) is taken outside the summation over the subchannels. This eliminates the bottleneck on achievable rates, caused by the per-subchannel constraints, and the rate region obtained by inter-subchannel cooperative encoding always contains that obtained by intra-subchannel cooperation. The rate regions achievable by both strategies will be compared for some sample fading scenarios, in Section IV.

\section{B. Half-duplex Cooperative Encoding}

The two cooperative OFDMA models proposed so far assumed full-duplex operation in each subchannel. However, in practice, due to the vast difference between the transmitted and received signal strengths, it is not possible to transmit and receive simultaneously on the same band. Hence, in this section, we define a more practical orthogonal cooperative encoding strategy, with half-duplex operation in each subchannel. The available subchannels are first divided into three sets, i.e., $\mathcal{I} \triangleq\{1, \ldots, N\}=\left\{\mathcal{I}_{1}, \mathcal{I}_{2}, \mathcal{I}_{3}\right\}$. On subchannels $i \in \mathcal{I}_{1}$, user 1 transmits, while user 2 and the receiver listen, on subchannels $i \in \mathcal{I}_{2}$, user 2 transmits while user 1 and the receiver listen, and on $i \in \mathcal{I}_{3}$, both users transmit simultaneously, to obtain coherent combining gain, while only the receiver listens. The encoding is done across subchannels, allowing the messages received in the previous block to be repartitioned in the next block. The achievable rate region for the half-duplex cooperative encoding strategy is described in the following theorem.

Theorem 2: For a two user cooperative OFDMA channel which employs half-duplex cooperative encoding, an achievable rate region is given by the closure of the convex hull of all rate pairs $\left(R_{1}, R_{2}\right)$, with $R_{s}=R_{1}+R_{2}$ satisfying

$$
\begin{aligned}
& R_{1}<\sum_{i \in \mathcal{I}_{1}} E\left[C\left(\frac{s_{12}^{(i)} p_{12}^{(i)}}{s_{12}^{(i)} p_{10}^{(i)}+1}\right)\right]+\sum_{i \in\left\{\mathcal{I}_{1}, \mathcal{I}_{3}\right\}} E\left[C\left(s_{10}^{(i)} p_{10}^{(i)}\right)\right] \\
& R_{2}<\sum_{i \in \mathcal{I}_{2}} E\left[C\left(\frac{s_{21}^{(i)} p_{21}^{(i)}}{s_{21}^{(i)} p_{20}^{(i)}+1}\right)\right]+\sum_{i \in\left\{\mathcal{I}_{2}, \mathcal{I}_{3}\right\}} E\left[C\left(s_{20}^{(i)} p_{20}^{(i)}\right)\right], \\
& R_{s}<\min \left\{\sum_{i \in \mathcal{I}_{1}} E\left[C\left(s_{10}^{(i)}\left(p_{10}^{(i)}+p_{12}^{(i)}\right)\right)\right]\right. \\
& +\sum_{i \in \mathcal{I}_{2}} E\left[C\left(s_{20}^{(i)}\left(p_{20}^{(i)}+p_{21}^{(i)}\right)\right)\right] \\
& +\sum_{i \in \mathcal{I}_{3}} E\left[C\left(s_{10}^{(i)} p_{1}^{(i)}+s_{20}^{(i)} p_{2}^{(i)}+2 \sqrt{s_{10}^{(i)} s_{20}^{(i)} p_{U_{1}}^{(i)} p_{U_{2}}^{(i)}}\right)\right]
\end{aligned}
$$




$$
\begin{aligned}
& \sum_{i \in \mathcal{I}_{1}} E\left[C\left(\frac{s_{12}^{(i)} p_{12}^{(i)}}{s_{12}^{(i)} p_{10}^{(i)}+1}\right)+C\left(s_{10}^{(i)} p_{10}^{(i)}\right)\right] \\
& +\sum_{i \in \mathcal{I}_{2}} E\left[C\left(\frac{s_{21}^{(i)} p_{21}^{(i)}}{s_{21}^{(i)} p_{20}^{(i)}+1}\right)+C\left(s_{20}^{(i)} p_{20}^{(i)}\right)\right] \\
& \left.+\sum_{i \in \mathcal{I}_{3}} E\left[C\left(s_{10}^{(i)} p_{10}^{(i)}+s_{20}^{(i)} p_{20}^{(i)}\right)\right]\right\} .
\end{aligned}
$$

Proof: The proof relies on the key observation that our proposed half-duplex scheme may be viewed as a special case of inter-subchannel cooperative encoding, with $p_{20}^{(i)}=p_{21}^{(i)}=$ $p_{U_{1}}^{(i)}=p_{U_{2}}^{(i)}=0$ for $i \in \mathcal{I}_{1}, p_{10}^{(i)}=p_{12}^{(i)}=p_{U_{1}}^{(i)}=p_{U_{2}}^{(i)}=0$ for $i \in \mathcal{I}_{2}$ and $p_{12}^{(i)}=p_{21}^{(i)}=0$ for $i \in \mathcal{I}_{3}$. Substituting these power levels in (23)-(25), we obtain the desired result.

\section{Simulation Results}

In this section we evaluate the achievable rate regions (23)-(25) for inter-subchannel cooperative encoding and (20)(22) for intra-subchannel cooperative encoding, and compare them with the capacity region of a non-cooperative OFDMA system, as well as the achievable rate region of our half-duplex cooperative encoding strategy (32)-(34). We assume that, for all four protocols, the users are able to allocate their total power across subchannels and codewords, and the achievable rate regions are generated by taking the convex hull over all valid power allocation policies. Note however that adaptive power allocation in terms of instantaneous fading states is not considered, the power assigned to each subchannel and codeword remains the same throughout the transmission. The total power of each user and the noise variances are both set to unity (except for Figure 5 where SNR is varied). Three subchannels were used in the simulations.

In Figure 3 the achievable rate/capacity regions are generated for fading scenario 1 , where we choose channel gains from independent Rayleigh distributions, the means of which are shown in the figure. While, for half-duplex cooperation with an arbitrary number of subchannels and arbitrary average channel qualities, it is not easy to find the optimal fixed subchannel allocation policy to the half-duplex operations of the users, in our particular example we can simply set $\mathcal{I}_{1}=\{1\}, \mathcal{I}_{2}=\{2\}$ and $\mathcal{I}_{3}=\{3\}$, which is in fact optimal among all fixed subchannel allocations. We see that the single user achievable rates for both cooperative strategies are similar, but the gap between the achievable rates of the two policies increase near the sum rate point. This can be explained as follows: the cooperative links on the first two subchannels are better on average, while the direct link is better on the third. Therefore, although the term $C_{s}^{(i)}$ is not very restrictive on the single user rates (20)-(21), it is restrictive for the sum rate (22). Since inter-subchannel cooperative coding can assign powers to cooperative codewords so that it mostly uses the third subchannel to send the cooperative codeword $U$, and the first two for cooperation among users, its advantage near the sum rate point is more pronounced. Under these channel conditions, we see that sum rates for both cooperative strategies are always higher than non-cooperative OFDMA while the sum rate of half-duplex cooperative encoding is higher than that of the intra-subchannel cooperative strategy

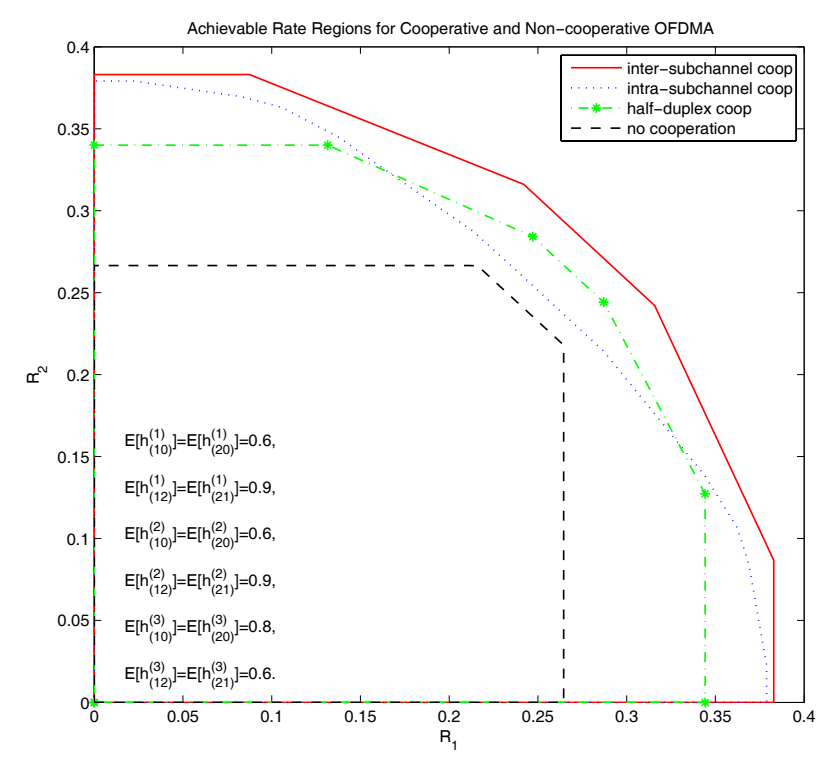

Fig. 3. Achievable rate regions for fading scenario 1. The inter-user links are stronger on subchannels 1 and 2, and direct links are stronger on subchannel 3. Subchannel allocation for half-duplex setting is set to the optimal strategy.

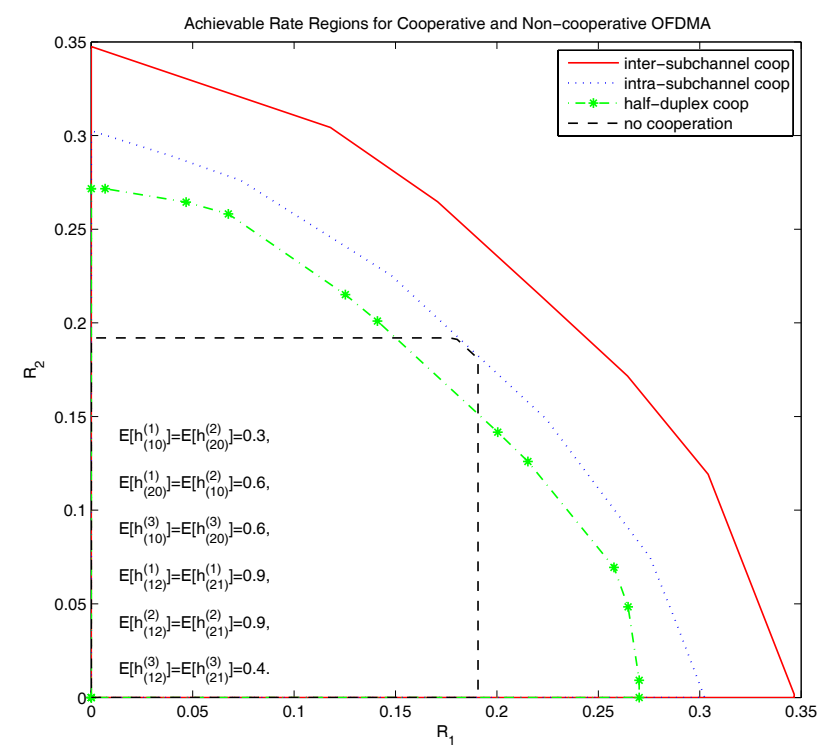

Fig. 4. Achievable rate regions for fading scenario 2. Direct link gains on subchannels 1 and 2 are asymmetrical, and on the average worse than inter-user-link gains. On subchannel 3, direct links are stronger than interuser links. Subchannel allocation for half-duplex setting is set to the optimal strategy.

since the direct links are stronger over the first and second subchannels and the cooperative link is stronger over the third subchannel, and with our selection of $\mathcal{I}_{1}, \mathcal{I}_{2}$ and $\mathcal{I}_{3}$, the half-duplex cooperative encoding uses subchannels 1 and 2 to create common information, and subchannel 3 for direct transmission to obtain coherent combining gain.

In Figure 4, we switch to the Rayleigh fading scenario 2, and the gap among the two cooperative policies becomes more apparent. Note that the direct link gain of user 1 is worse than user 2 on subchannel 1 , and vice versa on subchannel 2 . Therefore, the rate gains achievable in those subchannels are 


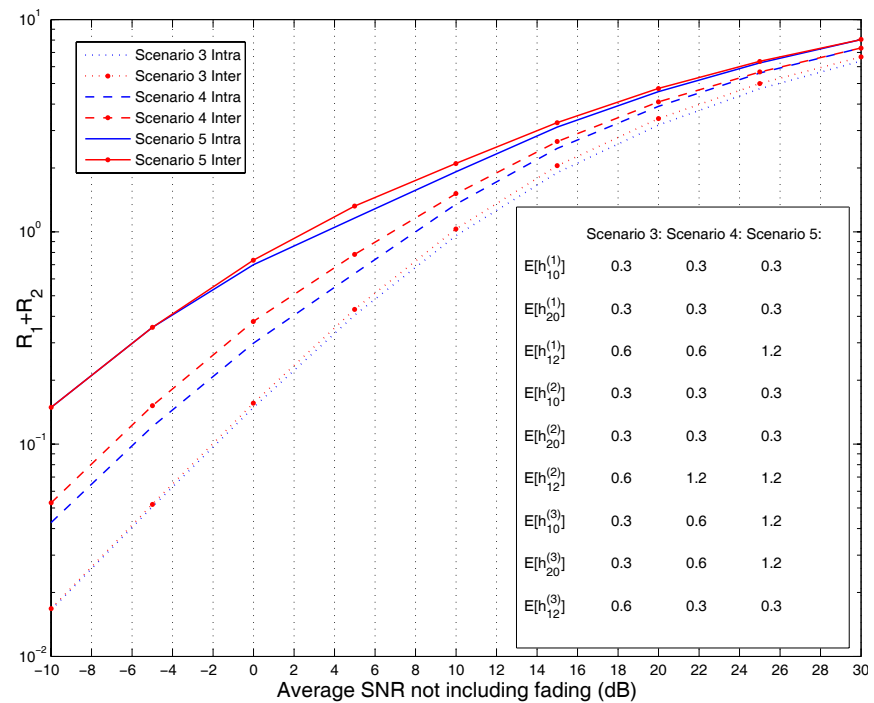

Fig. 5. Comparison of sum rates achievable by intra and inter-subchannel cooperative encoding, as a function of SNR. The performance depends on relative average strengths of fading coefficients on subchannels, rather than SNR: in scenarios 4 and 5 , there is nearly $2 \mathrm{~dB}$ gain at low and moderate SNR values respectively, in the symmetric scenario 3 where no subchannel provides a relative advantage to either policy, inter-subchannel cooperation only slightly outperforms intra-subchannel cooperation over almost all SNR values.

hindered by the per-subchannel constraints of intra-subchannel cooperation, and in fact around the sum rate point it achieves rates only slightly better than non-cooperative OFDMA, while inter-subchannel cooperation still gives large gains. The sum rate of half-duplex cooperation, which uses the same optimal channel assignment as above, is less than both full-duplex cooperative strategies due to the fact that the achievable rate of the half-duplex cooperative encoding relies heavily on the direct link gains on subchannel 3, that is relatively weak for this fading scenario.

In Figure 5, we provide further simulation results comparing the sum rates achievable by intra and inter-subchannel cooperation, as a function of the average transmit signal power to noise power ratio, for three other fading scenarios (3-5) described in the respective figures, where we choose $E\left[h_{12}^{(i)}\right]=E\left[h_{21}^{(i)}\right]$. We observe the relative behavior for varying SNR to depend highly on the fading statistics, but especially when the fading is asymmetric, the gain is more pronounced for low and moderate SNR values.

\section{CONCLUSION}

In this paper we introduced a two user cooperative OFDMA system, and we proposed two full-duplex encoding strategies: intra-subchannel cooperative encoding and inter-subchannel cooperative encoding, based on BMSE. We derived rate region expressions for both encoding strategies and showed that re-partitioning and re-encoding of the cooperative messages across subchannels, i.e., inter-subchannel cooperative encoding, is always superior to intra-subchannel cooperative encoding, and provides significant rate gains. We further proposed a half-duplex implementation of cooperation based on intersubchannel cooperative encoding, and showed that despite its orthogonal structure, it too may outperform the full-duplex intra-subchannel cooperation under some cases. Achievable rates for all three proposed scenarios show that it is very advantageous to make use of overheard information, especially taking into account the added diversity created by the OFDMA subchannels.

\section{REFERENCES}

[1] S. Bakım and O. Kaya, "Achievable rates for two user cooperative OFDMA," in Proc. 2010 IEEE Global Commun. Conf., pp. 1-5.

[2] A. Carleial, "Multiple-access channels with different generalized feedback signals," IEEE Trans. Inf. Theory, vol. 28, no. 6, pp. 841-850, 1982.

[3] F. M. J. Willems, E. C. van der Meulen, and J. P. M. Schalkwijk, "An achievable rate region for the multiple access channel with generalized feedback," in Proc. 1983 Allerton Conf.

[4] A. Sendonaris, E. Erkip, and B. Aazhang, "User cooperation diversitypart I: system description,” IEEE Trans. Commun., vol. 51, no. 11, pp. 1927-1938, Nov. 2003.

[5] J. Lin and A. Stefanov, "Coded cooperation for OFDM systems," in Proc. 2005 International Conf. Wireless Netw., Commun. Mobile Comput., vol. 1, pp. 7-10.

[6] B. Can, H. Yomo, and E. D. Carvalho, "Hybrid forwarding scheme for cooperative relaying in OFDM based networks," in Proc. 2006 IEEE International Conf. Commun., pp. 4520-4525.

[7] L. Dai, B. Gui, and L. Cimini, "Selective relaying in OFDM multihop cooperative networks," in Proc. 2007 IEEE Wireless Commun. Netw. Conf., pp. 963-968.

[8] L. Weng and R. D. Murch, "Cooperation strategies and resource allocations in multiuser OFDMA systems," IEEE Trans. Veh. Technol., vol. 58, no. 5, pp. 2331-2342, June 2009.

[9] W. Shim, Y. Han, and S. Kim, "Fairness-aware resource allocation in a cooperative OFDMA uplink system," IEEE Trans. Veh. Technol., vol. 59, no. 2, pp. 932-939, Feb. 2010.

[10] S. Bakım, "Cooperative strategies, achievable rates and resource allocation for OFDMA channels," Ph.D. thesis, Işık University, Istanbul, Turkey, July 2011.

[11] T. Cover and J. Thomas, Elements of Information Theory. Wiley, 1991. 